\title{
SERIES INVOLVING PRODUCTS OF TWO E-FUNCTIONS
}

\author{
by ARUN VERMA $†$
}

(Received 7 August, 1963)

1. Introduction. In $1958 \mathrm{Ragab}$ [3] deduced the sums of certain infinite series involving a product of two $E$-functions in terms of $E$-functions. MacRobert [2] gave a very simple alternative method for proving the results of Ragab. Later, Ragab $[4,5]$ in 1962 used a method similar to the one given by MacRobert to deduce a number of summations involving products of $E$-functions. In this paper, some more general summations of $E$-functions, which contain Ragab's results as special cases, are given. It may be mentioned that all the series summed run from $n=-\infty$ to $+\infty$ instead of $n=0$ to $+\infty$.

The $E$-function is defined by the contour integral

$$
E\left[p ; \alpha_{r}: q ; \rho_{s}: z\right]=\frac{1}{2 \pi i} \int \frac{\Gamma[\zeta] \prod_{r=1}^{p} \Gamma\left[\alpha_{r}-\zeta\right]}{\prod_{s=1}^{q} \Gamma\left[\rho_{s}-\zeta\right]} z^{\zeta} d \zeta
$$

provided that $|\operatorname{amp} z|<\pi$. The integral is taken upwards along the $\eta$-axis, with loops, if necessary, to ensure that the pole at the origin lies to the left and the poles at $\alpha_{1}, \alpha_{2}, \ldots, \alpha_{p}$ to the right of the contour. Zero and negative values of $\alpha_{r}$ and $\rho_{s}$ are excluded and the $\alpha_{r}$ must not differ by integral values. When $p<q+1$ the contour is bent to the left at both ends, and if $p \geqq q+1$, the formula is valid for $|\operatorname{amp} z|<\frac{1}{2}(p-q+1) \pi$. In what follows the contour integrals are all taken over the above contour.

Further, $\Delta_{n}[p ; \alpha]$ denotes the sequence of $n$ terms

$$
\frac{\alpha}{p}, \frac{\alpha+1}{p}, \ldots, \frac{\alpha+n-1}{p},
$$

but when $n=p$ we write simply $\Delta[p ; \alpha]$ instead of $\Delta_{p}[p ; \alpha]$.

Lastly, a bilateral hypergeometric series of order $p$ is defined by

$$
{ }_{p} H_{p}\left[\begin{array}{l}
\left.a_{1}, a_{2}, \ldots, a_{p} ; x\right] \\
b_{1}, b_{2}, \ldots, b_{p}
\end{array}\right]=\sum_{n=-\infty}^{\infty} \frac{\left[a_{1}\right]_{n}\left[a_{2}\right]_{n} \ldots\left[a_{p}\right]_{n} x^{n}}{\left[b_{1}\right]_{n}\left[b_{2}\right]_{n} \ldots\left[b_{p}\right]_{n}} \quad(|x|=1),
$$

provided that $\operatorname{Re}\left[\sum\left(b_{p}\right)-\sum\left(a_{p}\right)-1\right]>0$, if $x=+1$, and $\operatorname{Re}\left[\sum\left(b_{p}\right)-\sum\left(a_{p}\right)\right]>0$, if $x=-1$; here $[\alpha]_{n}=\alpha(\alpha+1)(\alpha+2) \ldots(\alpha+n-1),[\alpha]_{0}=1$, and $[\alpha]_{-n}=(-)^{n} /[1-\alpha]_{n}$.

† This work has been done under a fellowship of the Council of Scientific and Industrial Research, India. 
2. We shall prove the following summation formulae for the product of two $E$-functions: $\sum_{n=-\infty}^{\infty} \frac{m^{2 n}}{[\gamma]_{n}[\delta]_{n}} E\left[\begin{array}{c}\alpha, 1-\alpha, \Delta[m ; \gamma+d-1], \Delta[m ; \delta+d-1]: z \\ \Delta[m ; d-n]\end{array}\right]$

$$
\begin{aligned}
& \times E\left[\begin{array}{c}
\beta, 1-\beta, \Delta[m ; \gamma+g-1], \Delta[m ; \delta+g-1]: e^{i n} z \\
\Delta[m ; g-n]
\end{array}\right]
\end{aligned}
$$

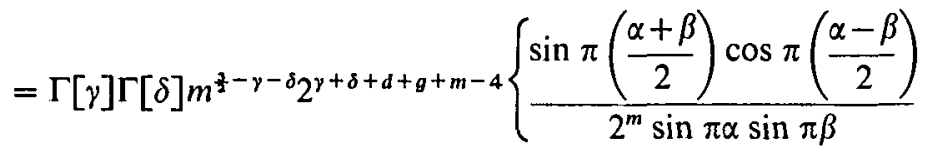

$$
\begin{aligned}
& \times E\left[\begin{array}{rrr}
\frac{1}{2}(1+\alpha-\beta), \frac{1}{2}(1+\beta-\alpha), \frac{1}{2}(\alpha+\beta), \frac{1}{2}(1-\alpha-\beta), & \Delta_{m}[2 m ; \gamma+\delta+d+g-3], \\
& \Delta_{m}[2 m ; \gamma+\delta+d+g-3+m]: \frac{e^{i \pi} z^{2}}{4^{m+1}} \\
\frac{1}{2} &
\end{array}\right] \\
& -\frac{2}{z} \frac{\cos \pi\left(\frac{\alpha+\beta}{2}\right) \sin \pi\left(\frac{\alpha-\beta}{2}\right)}{\sin \pi \alpha \sin \pi \beta} \\
& \times E\left[\begin{array}{ccc}
\frac{1}{2}(2+\alpha-\beta), \frac{1}{2}(2+\beta-\alpha), \frac{1}{2}(1+\alpha+\beta), \frac{1}{2}(3-\alpha-\beta), \Delta_{m}[2 m ; \gamma+\delta+d+g-3+m], \\
\frac{3}{2} & \Delta_{m}[2 m ; \gamma+\delta+d+g-3+2 m]: \frac{e^{i \pi} z^{2}}{4^{m+1}}
\end{array}\right],
\end{aligned}
$$

provided that $\operatorname{Re}(\beta)>0, \operatorname{Re}(\gamma+\delta+d+g)>3,|\operatorname{amp} z|<\frac{1}{2}(m+1) \pi$.

$$
\begin{aligned}
& \sum_{n=-\infty}^{\infty} \frac{(-)^{n}[\varepsilon]_{n}}{[\delta]_{n}} E\left[\begin{array}{c}
\alpha, 1-\alpha, \Delta[m ; \delta-\theta]: z \\
\Delta[m ; 1-\theta-n]
\end{array}\right] E\left[\begin{array}{c}
\beta, 1-\beta, \Delta[m ; \gamma-\varepsilon]: e^{i \pi} z \\
\Delta[m ; \gamma+n]
\end{array}\right] \\
& =2^{\delta-\varepsilon} \sqrt{\pi} \frac{\Gamma[\delta] \Gamma[1-\varepsilon]}{\Gamma[\delta-\varepsilon]} \times\left\{\frac{\sin \pi\left(\frac{\alpha+\beta}{2}\right) \cos \pi\left(\frac{\alpha-\beta}{2}\right)}{2 \sin \pi \alpha \sin \pi \beta}\right. \\
& \times E\left[\begin{array}{c}
\frac{1}{2}(1+\alpha-\beta), \frac{1}{2}(1+\beta-\alpha), \frac{1}{2}(\alpha+\beta), \frac{1}{2}(1-\alpha-\beta), \Delta_{m}[2 m ; \delta+\gamma-\varepsilon-\theta-1], \\
\Delta_{m}[2 m ; \delta+\gamma-\varepsilon-\theta-1+m]: \frac{e^{i \pi} z^{2}}{4} \\
\frac{1}{2}, \Delta_{m}[2 m ; \gamma-\theta], \Delta_{m}[2 m ; \gamma-\theta+m]
\end{array}\right] \\
& -\frac{\cos \pi\left(\frac{\alpha+\beta}{2}\right) \sin \pi\left(\frac{\alpha-\beta}{2}\right)}{z \sin \pi \alpha \sin \pi \beta} \\
& \times E\left[\begin{array}{c}
\frac{1}{2}(2+\alpha-\beta), \frac{1}{2}(2+\beta-\alpha), \frac{1}{2}(1+\alpha+\beta), \frac{1}{2}(3-\alpha-\beta), \Delta_{m}[2 m ; \delta+\gamma-\varepsilon-\theta-1+m], \\
\Delta_{m}[2 m ; \delta+\gamma-\varepsilon-\theta-1+2 m]: \frac{e^{i \pi} z^{2}}{4} \\
\frac{3}{2}, \Delta_{m}[2 m ; \gamma-\theta+m], \Delta_{m}[2 m ; \gamma-\theta+2 m]
\end{array}\right],
\end{aligned}
$$


provided that $\operatorname{Re}(\beta)>0, \operatorname{Re}(\delta+\gamma-\varepsilon-\theta)>1, \operatorname{Re}(\delta-\varepsilon)>1, \mid$ amp $z \mid<\pi / 2$.

$$
\begin{aligned}
& \sum_{n=-\infty}^{\infty} \frac{[1+a / 2]_{n}[b]_{n}[c]_{n}}{[a / 2]_{n}[1+a-b]_{n}[1+a-c]_{n}} E\left[\begin{array}{cc}
\alpha, 1-\alpha, \Delta[m ; a+e-c], \Delta[m ; a+e-b]: z \\
\Delta[m ; e-n], & \Delta[m ; a+e+n]
\end{array}\right] \\
& \times E\left[\begin{array}{cc}
\beta, 1-\beta, \Delta[m ; a+h-c], \Delta[m ; a+h-b]: e^{i \pi} z \\
\Delta[m ; h-n], \quad \Delta[m ; a+h+n]
\end{array}\right] \\
& =\left(\frac{m}{2}\right)^{b+c-a} \frac{\Gamma[1-b] \Gamma[1-c] \Gamma[1+a-b] \Gamma[1+a-c]}{\Gamma[1+a] \Gamma[1-a] \Gamma[1+a-b-c] \sin \pi \alpha \sin } \frac{\sqrt{\pi}}{\pi \beta}\left\{\sin \pi\left(\frac{\alpha+\beta}{2}\right) \cos \pi\left(\frac{\alpha-\beta}{2}\right)\right. \\
& \times E\left[\begin{array}{c}
\frac{1}{2}(1+\alpha-\beta), \frac{1}{2}(1+\beta-\alpha), \frac{1}{2}(\alpha+\beta), \frac{1}{2}(1-\alpha-\beta), \Delta_{m}[2 m ; 2 a+h+e-b-c-1], \\
\Delta_{m}[2 m ; 2 a+h+e-b-c+m-1]: \frac{e^{i \pi} z^{2}}{4} \\
\frac{1}{2}, \Delta_{m}[2 m ; a+h+e-1], \Delta_{m}[2 m ; a+h+e-1+m]
\end{array}\right] \\
& -\frac{2}{z} \cos \pi\left(\frac{\alpha+\beta}{2}\right) \sin \pi\left(\frac{\alpha-\beta}{2}\right) \\
& \times E\left[\begin{array}{c}
\frac{1}{2}(2+\alpha-\beta), \frac{1}{2}(2+\beta-\alpha), \frac{1}{2}(1+\alpha+\beta), \frac{1}{2}(3-\alpha-\beta), \\
\Delta_{m}[2 m ; 2 a+h+e-b-c-1+m], \Delta_{m}[2 m ; 2 a+h+e-b-c-1+2 m]: \frac{e^{i \pi} z^{2}}{4} \\
\frac{3}{2}, \Delta_{m}[2 m ; a+h+e-1+m], \Delta_{m}[2 m ; 2 a+h+e-1+2 m]
\end{array}\right],
\end{aligned}
$$

provided that $\operatorname{Re}(\beta)>0, \operatorname{Re}(a+h+e)>\operatorname{Re}(2 a+h+e-b-c)>1, \mid$ amp $z \mid<\pi / 2$.

$$
\begin{gathered}
\sum_{n=-\infty}^{\infty} \frac{m^{2 n}}{[\gamma]_{n}[\mu]_{n}} E\left[\begin{array}{c}
\alpha, \beta, \Delta[m ; \gamma-\delta], \Delta[m ; \mu-\delta]: z \\
\theta, \Delta[m ; 1-\delta-n]
\end{array}\right] E\left[\begin{array}{c}
\theta-\alpha-\beta, \Delta[m ; \gamma-\varepsilon], \Delta[m ; \mu-\varepsilon]: z \\
\Delta[m ; 1-\varepsilon-n]
\end{array}\right] \\
=(2 \pi)^{\frac{1}{2}(m-1)} m^{\frac{3}{2}-\gamma-\mu} \frac{\Gamma[\gamma] \Gamma[\mu] \Gamma[\alpha] \Gamma[\beta] \Gamma[\theta-\alpha-\beta]}{\Gamma[\theta-\alpha] \Gamma[\theta-\beta]} \\
\times E\left[\begin{array}{c}
\theta-\alpha, \theta-\beta, \Delta[m ; \gamma+\mu-\delta-\varepsilon-1]: z \\
\theta
\end{array}\right],
\end{gathered}
$$

provided that $\operatorname{Re}(\gamma+\mu-\delta-\varepsilon)>1, \operatorname{Re}(\theta-\alpha-\beta)>0,|\operatorname{amp} z|<\frac{1}{2}(m+2) \pi$.

$$
\begin{gathered}
\sum_{n=-\infty}^{\infty} \frac{m^{2 n}}{[\gamma]_{n}[\mu]_{n}} E\left[\begin{array}{c}
\beta, \Delta[m ; \gamma-\delta], \Delta[m ; \mu-\delta]: z \\
\Delta[m ; 1-\delta-n]
\end{array}\right] E\left[\begin{array}{c}
\theta, \Delta[m ; \gamma-\varepsilon], \Delta[m ; \mu-\varepsilon]: z \\
\Delta[m ; 1-\varepsilon-n]
\end{array}\right] \\
=(2 \pi)^{\frac{1}{2(m-1)}} m^{\frac{3}{2-\mu-\gamma}} \frac{\Gamma[\mu] \Gamma[\gamma] \Gamma[\beta] \Gamma[\theta]}{\Gamma[\beta+\theta]} E[\beta+\theta, \Delta[m ; \gamma+\mu+\delta-\varepsilon-1]:: z],
\end{gathered}
$$

provided that $\operatorname{Re}(\gamma+\mu-\delta-\varepsilon)>1$ and $|\operatorname{amp} z|<\frac{1}{2}(m+2) \pi$.

$\sum_{n=-\infty}^{\infty} \frac{m^{2 n}}{[\gamma]_{n}[\mu]_{n}} E\left[\begin{array}{c}\alpha, \beta, \Delta[m ; \gamma-\delta], \Delta[m ; \mu-\delta]: z \\ \alpha+\beta+\frac{1}{2}, \Delta[m ; 1-\delta-n]\end{array}\right] E\left[\begin{array}{c}\frac{1}{2}-\alpha, \frac{1}{2}-\beta, \Delta[m ; \mu-\varepsilon], \Delta[m ; \gamma-\varepsilon]: z \\ \frac{3}{2}-\alpha-\beta, \Delta[m ; 1-\varepsilon-n]\end{array}\right]$ 


$$
\begin{gathered}
=(2 \pi)^{\frac{1}{2}(m-1)} m^{\frac{2}{2}-\gamma-\mu} \pi^{-\frac{2}{2}} \cos \pi(\alpha-\beta) \Gamma[\alpha] \Gamma[\beta] \Gamma[\mu] \Gamma[\gamma] \Gamma\left[\frac{1}{2}-\alpha\right] \Gamma\left[\frac{1}{2}-\beta\right] \\
\times E\left[\begin{array}{c}
\frac{1}{2}, \alpha-\beta+\frac{1}{2}, \beta-\alpha+\frac{1}{2}, \Delta[m ; \gamma+\mu-\delta-\varepsilon-1]: z \\
\alpha+\beta+\frac{1}{2}, \frac{3}{2}-\alpha-\beta
\end{array}\right],
\end{gathered}
$$

provided that $\operatorname{Re}(\gamma+\mu-\delta-\varepsilon)>1$ and $|\operatorname{amp} z|<\frac{1}{2}(m+2) \pi$.

In general, to prove these results, we substitute the contour integrals for the $E$-functions from (1) in the left-hand side of the formula, change the order of summation and integration and sum the inner bilateral hypergeometric series by one of the well-known theorems. Then we use (1), once again, to rewrite the value of the inner contour integral in terms of an $E$ function. Finally, to evaluate this contour integral involving an $E$-function, we use certain known results of Ragab [4,5] and MacRobert [2].

We illustrate below, in detail, the above method in the case of summation formula (2).

Proof of (2). On substituting the contour integral (1) for the two $E$-functions on the lefthand side of (2), using the formula

$$
\Gamma[m z]=(2 \pi)^{\frac{1}{(1-m)} m^{m z-\frac{1}{2}}} \prod_{k=0}^{m-1} \Gamma\left[z+\frac{k}{m}\right],
$$

and then changing the order of summation and integration, which is justified when

$$
\operatorname{Re}(\gamma+\delta+d+g)>3
$$

the left-hand side of (2) becomes

$$
\begin{gathered}
\frac{1}{2 \pi i} \int_{c_{1}} \frac{\Gamma[\xi] \Gamma[\alpha-\xi] \Gamma[1-\alpha-\xi] \prod_{k=0}^{m-1} \Gamma\left[\frac{\gamma+d-1+k}{m}-\xi\right] \prod_{k=0}^{m-1} \Gamma\left[\frac{\delta+d-1+k}{m}-\xi\right] z^{\xi}}{\prod_{k=0}^{m-1} \Gamma\left[\frac{d+k}{m}-\xi\right]} \\
\times \frac{1}{2 \pi i} \int_{c_{2}} \frac{\Gamma[\zeta] \Gamma[\beta-\zeta] \Gamma[1-\beta-\zeta] \prod_{k=0}^{m-1} \Gamma\left[\frac{\gamma+g-1+k}{m}-\zeta\right] \prod_{k=0}^{m-1} \Gamma\left[\frac{\delta+g-1+k}{m}-\zeta\right] z^{\zeta} e^{i \pi \zeta}}{\prod_{k=0}^{m-1} \Gamma\left[\frac{g+k}{m}-\zeta\right]} \\
\times{ }_{2} H_{2}\left[\begin{array}{c}
1-d+m \xi, 1-g+m \zeta ; 1 \\
\gamma, \delta
\end{array}\right] d \xi d \zeta .
\end{gathered}
$$

Summing the inner bilateral hypergeometric series by a result due to Dougall $[1 ; 1.3]$ and then using (8) and finally (1) once again, we find that the above expression equals

$$
\begin{aligned}
&(2 \pi)^{\frac{1}{2}(m-1)} m^{\frac{2}{2}-\gamma-\delta} \Gamma[\gamma] \Gamma[\delta] \cdot \frac{1}{2 \pi i} \int_{C_{1}} \Gamma[\xi] \Gamma[\alpha-\xi] \Gamma[1-\alpha-\xi] z^{\xi} \\
& \times E\left[\beta, 1-\beta, \Delta[m ; \gamma+\delta+d+g-3-m \xi]:: e^{i \pi} z\right] d \xi .
\end{aligned}
$$

Evaluating this contour integral by a result due to Ragab [5; eqn. (10)], we get the required result (2). 
If we take $m=\delta=d=g=1$ in (2), it reduces to a result which is equivalent to one of Ragab [5; eqn. (12)].

3. To prove (3), the inner bilateral series as mentioned above comes out to be again a ${ }_{2} \mathrm{H}_{2}(+1)$, which can be summed as in (2) and the contour integral used finally is due to Ragab [5; eqn. (10)].

To prove (5), the inner bilateral hypergeometric series ${ }_{5} H_{5}(+1)$ is summable by a result due to Bailey $[1 ;(2.3)]$ and the integral used in the final stage is due to Ragab [5; eqn. (10)].

To derive (4), (6) and (7), the inner bilateral hypergeometric series summed are in all cases $\mathrm{a}_{2} \mathrm{H}_{2}(+1)$ and the contour integrals used in the final steps are due to MacRobert [2; eqns (5) and (6)] and Ragab [4; eqn. (8)] respectively.

In the above expressions, if we take one of the denominator parameters in the coefficients of the $E$-function to be unity, the summation reduces to one from $n=0$ to $+\infty$, which again yields generalisations of Ragab's result.

4. In this section, we briefly discuss the convergence conditions of the various summation formulae given in $\$ 2$. For the sake of convenience, let us take all the parameters to be real and positive. The results for complex values follow by analytic continuation on writing the value of the $E$-functions in (2) in terms of a contour integral by the help of (1) and then using the asymptotic expansions of all gamma functions containing an $n$ for $n \rightarrow \infty$; we find that

$$
E\left[\begin{array}{c}
\alpha, 1-\alpha, \Delta[m ; \gamma+d-1], \Delta[m ; \delta+d-1]: z \\
\Delta[m ; d-n]
\end{array}\right]=O\left[e^{-n} m^{-n} n^{n-d+\frac{1}{2}}\right],
$$

for large values of $n$. Using (9) in (2), we deduce that the expansion converges under the stated conditions.

The convergence of the other expansions can be worked out on similar lines.

It may be remarked that the formulae derived in this paper are only a few representative ones. A huge variety of such summations can be deduced by using the known summation theorems of bilateral hypergeometric functions and different types of contour integrals involving $E$-functions and products of gamma functions.

My sincere thanks are due to Dr R. P. Agarwal for his help and suggestions during the preparation of this paper.

\section{REFERENCES}

1. W. N. Bailey, Series of hypergeometric type which are infinite in both directions, Quart. J. Math. (Oxford) 7 (1936), 105-115.

2. T. M. MacRobert, Integration of $E$-functions with respect to their parameters, Proc. Glasgow Math. Assoc. 4 (1959), 84-87.

3. F. M. Ragab, Expansions of an $E$-function in a series of products of $E$-functions, Proc. Glasgow Math. Assoc. 3 (1958), 194-195.

4. F. M. Ragab, Summation of a series of products of E-functions, Proc. Glasgow Math. Assoc. 5 (1962), 118-120. 222-230.

5. F. M. Ragab, Reihen von Produkten MacRobertsche E-functionen, Math. Z. 78 (1962),

\section{THE UNIVERSITY}

LUCKNOW, INDIA 